\title{
Surface characterization and parameter estimation for industrial vision system
}

\author{
S JAGDISH, PRABIR KUMAR BISWAS, JAYANTA MUKHERJEE \\ and B N CHATTERJI*
}

Department of Electronics and Electrical Communication Engineering, Indian Institute of Technology, Kharagpur 721 302, India

MS received 15 July 1994; revised 18 May 1995

\begin{abstract}
In this paper we have presented some geometric techniques to characterize and parametrize surfaces of industrial parts in range images. The surfaces are characterized to one of plane, sphere, cylinder and cone, because they form the majority of object surfaces in man-made industrial parts. The problem has been studied for two different situations. In the first case, a priori knowledge about the surface shape is assumed. In such a situation the problem of surface characterization reduces to that of surface parameter estimation. The standard deviations of the estimated parameters give a measure of uncertainty of characterizing a surface patch to one of the four surface types. In the second case, no a priori information regarding the shape of a surface is available. This includes partially visible surfaces also. To deal with such a situation, a fuzzy classifier is designed using the uncertainty values. The fuzzy classifier classifies the unknown surface patch (including partially visible surfaces) to one of the four surface types. Experimental results with synthetic range images are presented to highlight the distinctive features of our technique.
\end{abstract}

Keywords. Range image; geometric surface fitting; parameter estimation; fuzzy classification; occlusion.

\section{Introduction}

The major aim of any computer vision system is to recover useful information about the three dimensional world from huge image arrays of sensed values. In the past these image arrays were digital intensity images. At each point in an intensity image the brightness value encodes information about surface geometry, surface reflectance characteristics, surface texture, scene illumination, the distance from the camera to an object surface, the characteristics of the intervening medium and the camera characteristics. However, such images do not contain explicit information about depth.

In recent years digital range images (also known as $2 \frac{1}{2}-\mathrm{D}$ images) have become available owing to the development of various active and passive range-finding 
techniques. Range data are produced in the form of an array of numbers, where the numbers quantify the distance from the sensor focal plane to the object surface within the field of view along rays emanating from points on a regularly spaced grid. Thus the range images provide direct geometrical-information about the shape of visible surfaces: Therefore, the process of recognising objects by their shape is less difficult in range images than in intensity images. Such images find wide application in industrial automation such as assembly automation, automated inspection of industrial parts etc.

Surface characterization and parameter estimation are two of the major problems of computer vision utilising range images in industrial environment. The estimated surface parameters can be used efficiently by the matching process for object recognition and pose determination even in presence of many undefined or occluded surface regions. A number of approaches for surface characterization and parameter extraction have been reported in the literature. The optimization and eigenvalue analysis (Faugeras 1984) approach employs global analysis of 3-D or $2 \frac{1}{2}-\mathrm{D}$ (range) data. This approach is more general in the sense that it is applicable to all sorts of quadrics and planar surfaces. Characterization by quadric invariants (e.g. sign of curvatures) (Besl \& Jain 1984, 1988; Brady et al 1985) employs computation of differential properties of the surface at each surface point. This technique requires spatial ordering of surface points. Surfaces are characterized according to the signs of mean and Gaussian curvatures.

Though eigenvalues or quadric invariants are good discriminators in a mathematical sense, they are often very sensitive to noise and quantization errors (Han et al 1987). Moreover in man-made industrial parts, the majority of object surfaces belong to planar, spherical, cylindrical and conical surfaces (Bolle \& Cooper 1984). Hence, for such applications it is not necessary to work with general quadric surfaces but rather to deal with a narrow domain of surface types, such as plane, sphere, cylinder and cone. Hough transform (Muller \& Mohr 1984) or modified Hough transform (Lin \& Wee 1985) techniques have been used in such cases for surface characterization purposes.

Parametric estimation technique by Han et al (1987) calculates the geometric properties of a sufficient set of shapes, namely plane, sphere and cylinder, based upon an analysis of the surface normals. This approach uses a mixture of histogramming and parameter-fitting techniques to estimate surface parameters.

We have presented in this paper some new geometric techniques which use transformation invariant properties of surface normals and surface tangents to obtain the description of an unknown surface in terms of plane, sphere, cylinder or cone. This work presumes surfaces in range images are already segmented. Range image segmentation is not addressed here. A number of techniques for range image segmentation have been discussed by Biswas et al (1990). Unlike Han et al (1987) who use histogram analysis of surface normals to extract surface regions of a particular type, our technique uses mean and standard deviation of parameters characterizing different surfaces. The standard deviation of a parameter represents the measure of uncertainty of fitting a particular type of surface with respect to that parameter. The problem has been attempted for two different situations. In the first case, it has been assumed that a priori knowledge about the shape of the surface is available. In such situations, a surface of proper type is fitted to the set of points representing the surface patch and the parameters of the fitted surface are extracted. This strategy of selective surface fitting reduces the amount of computation. In the second case, when no a priori information regarding the shape of the unknown surface is available, the characterization of the surface is obtained in the 
form of a fuzzy membership vector. A fuzzy classifier is designed using the uncertainty measures which classifies an unknown surface to one of the surface types.

Geometric surface fitting and parameter estimation is discussed in $\S 2$. Section 3 describes the fuzzy classifier to classify an unknown surface. Occluded surface characterization is discussed in $\$ 4$.

\section{Surface characterization through geometric method}

The geometric method described in this section is guided by a priori information regarding the shape of a surface patch to fit an appropriate surface and extract the parameters of the fitted surface. The parameters extracted for each of planar, spherical, cylindrical and conical surfaces are the surface normal and the perpendicular distance from the origin for a plane, the centre coordinates and the radius for a sphere, the axis and the radius for a cylinder and the apex, the apex angle and the axis for a cone. The geometric method of surface fitting for these surfaces is facilitated from the existence of certain unique and transformation invariant properties of their surface normals and surface tangents as listed below:

Plane: All surface normals are parallel (to the normal to the plane).

Sphere: All surface normals are concurrent (at the centre of the sphere).

Cylinder: All surface normals are perpendicular to the axis of the cylinder.

Cone: All tangential planes to the surface intersect at a point (at the apex of the cone).

In the following discussion the input is taken to be a set of $M$ points in three dimensional space. The ith point is referred to as $X_{i} \equiv\left(x_{i}, y_{i}, z_{i}\right)$ or equivalently by $\mathbf{X}_{i}$, the vector joining the origin $(0,0,0)$ to $\left(x_{i}, y_{i}, z_{i}\right)$. The set of corresponding normals is taken to be $\left\{\mathbf{N}_{i} \mid i=1,2, \ldots, M\right\}$. The normal $\mathbf{N}_{i}$ at point $\mathbf{X}_{i}$ is computed by fitting a plane to the $5 \times 5$ neighbourhood of $X_{i}$ using least square error method (Taylor et al 1989).

\subsection{Characterization of a plane}

A plane is characterized by the direction of the normal to the plane and the perpendicular distance of the plane from the origin (refer figure 1). The estimate of the normal to the plane approximating the set of input points is given by

$$
\mathbf{N}_{\mathrm{av}}=\sum_{i=1}^{\boldsymbol{M}} \mathbf{N}_{i} /\left|\sum_{i=1}^{\boldsymbol{M}} \mathbf{N}_{i}\right|,
$$

where $\mathbf{N}_{i}$ is the unit normal vector at point $X_{i}$ and ' $\Sigma$ ' in (1) represents vector addition. The uncertainty in the estimation of the surface normal is given by the standard deviation $\sigma_{N}$ of $\mathrm{N}_{\mathrm{av}}$ where

$$
\boldsymbol{\sigma}_{N}^{2}=\frac{1}{M} \sum_{i=1}^{M}\left|\mathbf{N}_{i}-\mathbf{N}_{\mathrm{av}}\right|^{2}
$$

where $\mid \cdot$ represents the magnitude of a vector. The lesser the value of $\sigma_{N}$, the more reliable is the normal estimate. The perpendicular distance $d$ of the plane from the 


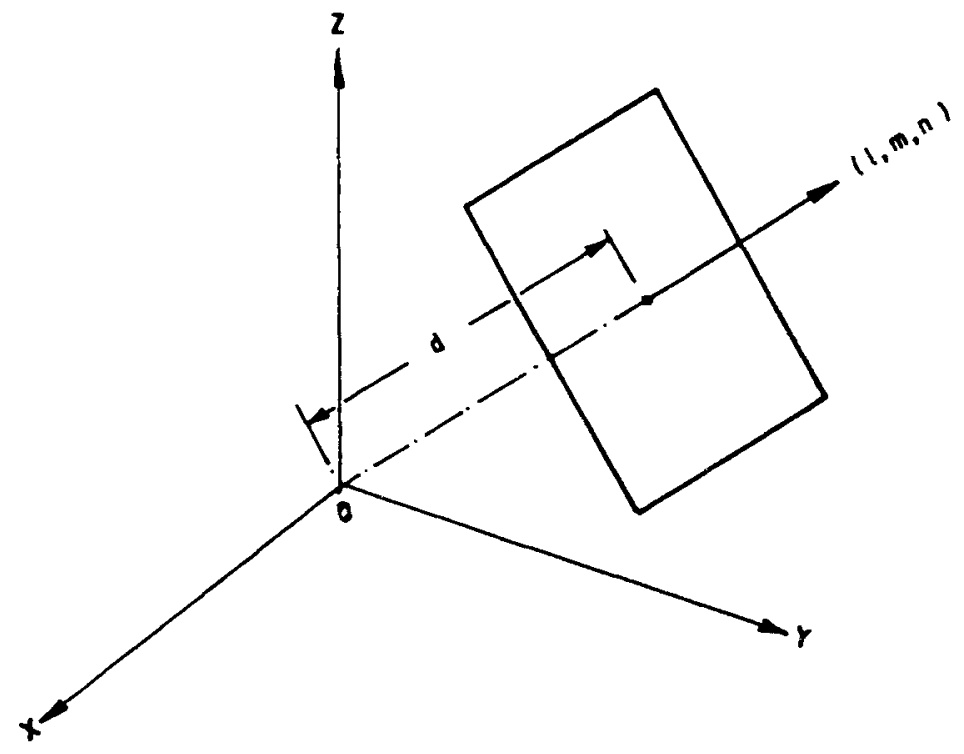

Figure 1. Direction of normal to a plane and the perpendicular distance of the plane from the origin.

origin and the standard deviation $\sigma_{d}$ of $d$ are given by

$$
\begin{aligned}
d & =\frac{1}{M} \sum_{i=1}^{M}\left(\mathbf{N}_{\mathrm{av}} \cdot \mathbf{X}_{i}\right), \\
\sigma_{d}^{2} & =\frac{1}{M} \sum_{i=1}^{M}\left(\mathbf{N}_{\mathrm{av}} \cdot \mathbf{X}_{i}-d\right)^{2} .
\end{aligned}
$$

The values of $\sigma_{N}$ and $\sigma_{d}$ together reflect the uncertainty of fitting a plane to the set of points.

\subsection{Characterization of a sphere}

Ideally, all the surface normals pass through the centre of a sphere. But in practice, owing to the presence of noise in the range data and to computational round-off errors, such a unique point cannot be determined. Rather, the majority of surface normals pass through a small neighbourhood of the centre of the sphere. So, for every pair of nonparallel normals $\mathbf{N}_{i}$ and $\mathbf{N}_{j}$ (refer figure 2), the position of the line perpendicular to both of them is determined (Shanti Narayan 1985). The length of the line segment intercepted between the two normal vectors also gives the shortest distance between them. The points of intersection $p_{i}$ and $p_{j}$ of this common perpendicular with the two normals are taken as two estimates of the centre of the sphere. The mean position of the centre $X_{c}$ and the standard deviation $\sigma_{x}$ are computed from these estimates.

Once the position of the centre is found, the radius $R$ can be estimated as

$$
R=\frac{1}{M} \sum_{i=1}^{M}\left|\mathbf{X}_{i}-\mathbf{X}_{c}\right|
$$




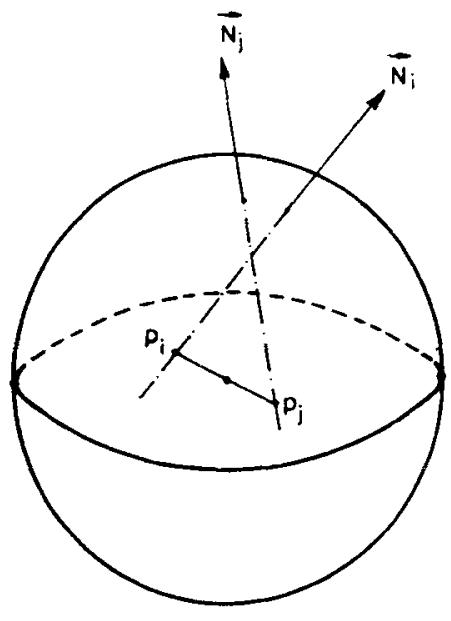

Figure 2. Estimation of the centre of a sphere.

The standard deviations of the centre $\sigma_{x}$ and the radius $\sigma_{R}$ together give a measure of uncertainty in fitting the sphere to the set of points.

\subsection{Characterization of a cylinder}

The parameters of interest in this case are the orientation and position of the axis and the radius of the cylinder. Every normal on the surface of a cylinder is perpendicular to the direction of the axis. Thus the cross-product of any two nonparallel surface normals gives an estimate of the orientation of the cylinder axis. The cross-products are calculated for all possible pairs of nonparallel surface normals. The average orientation of all these cross-products determines the candidate axis direction. Thus, $\forall i, j$ where $\mathbf{N}_{i}$ and $\mathbf{N}_{j}$ are nonparallel, the $k$ th estimate of axis orientation is given by the vector $\tau_{k}$ where

$$
\boldsymbol{\tau}_{\mathbf{k}}=\left(\mathbf{N}_{i} \times \mathbf{N}_{j}\right) /\left|\mathbf{N}_{i} \times \mathbf{N}_{j}\right|,
$$

From these estimates the average orientation $\tau_{\mathrm{av}}$ and the standard deviation $\sigma_{\tau}$ are computed. The position of the axis is determined by finding a point on the axis which is done in the following manner.

At each point $\mathbf{X}_{i}$, the plane on which $\mathbf{X}_{i}, \mathbf{N}_{i}$ and $\tau_{\mathrm{av}}$ lie is determined. The intersection of two such nonparallel planes (Shanti Narayan 1985) gives an estimate of the axis position (refer figure 3). From these estimates average estimate $\mathbf{X}_{\mathrm{av}}$ and standard deviation $\sigma_{x}$ are computed.

A point to be noted is that to get an estimate of the same point on the axis in all cases, the estimates are considered for the point at which the cylinder axis intersects the $x=0$ piane if axis is not parallel to that plane, else the $y=0$ plane if axis is not parallel to that plane, else the $z=0$ plane.

Once the orientation and the position of the axis are fixed, at each point $X_{i}$, the radius $R_{i}$ of the cylinder (refer figure 4 ) can be estimated as follows.

$$
R_{i}=\left|\mathbf{X}_{i}-\mathbf{X}_{\mathrm{av}}\right|^{*} \sin \theta=\left|\left(\mathbf{X}_{i}-\mathbf{X}_{\mathrm{av}}\right) \times \tau_{\mathrm{av}}\right|
$$

From different estimates of $R_{i}$ we compute $R_{\mathrm{av}}$ and $\sigma_{R}$.

The standard deviations of the axis orientation $\sigma_{\tau}$, axis position $\sigma_{X}$ and the radius $\sigma_{R}$ together give a measure of the uncertainty of fit. 


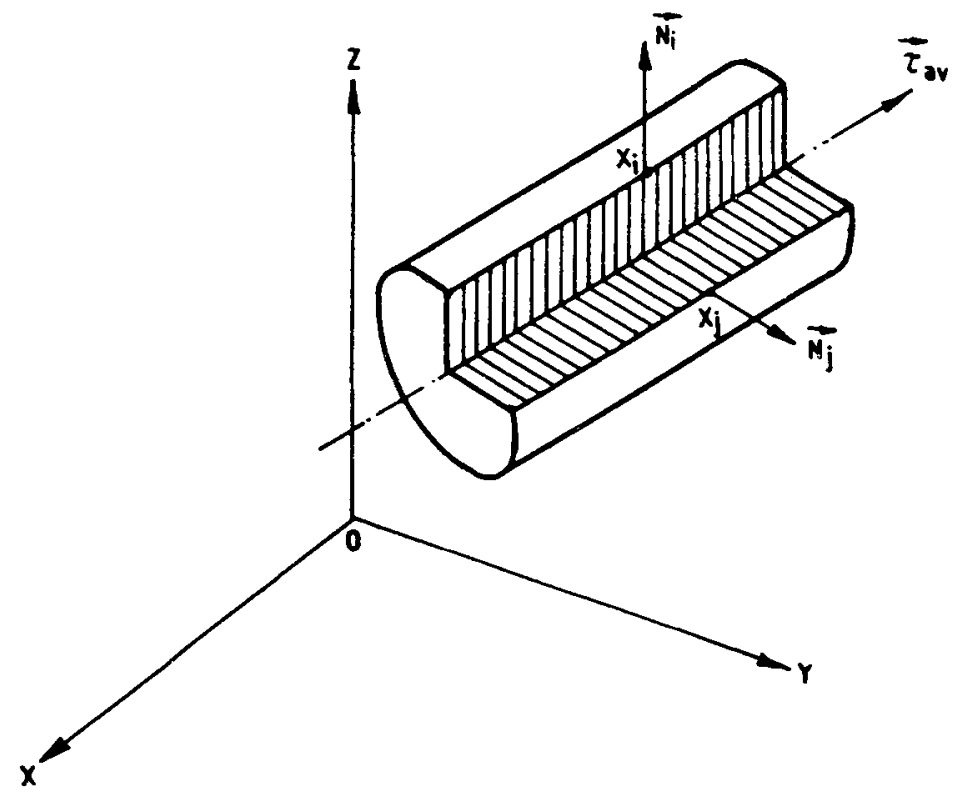

Figure 3. Estimation of the axis position of a cylinder.

\subsection{Characterization of a cone}

A cone is described by the position of its apex, orientation of its axis and the apex angle. Ideally the point of intersection of all tangential planes specifies the apex point.

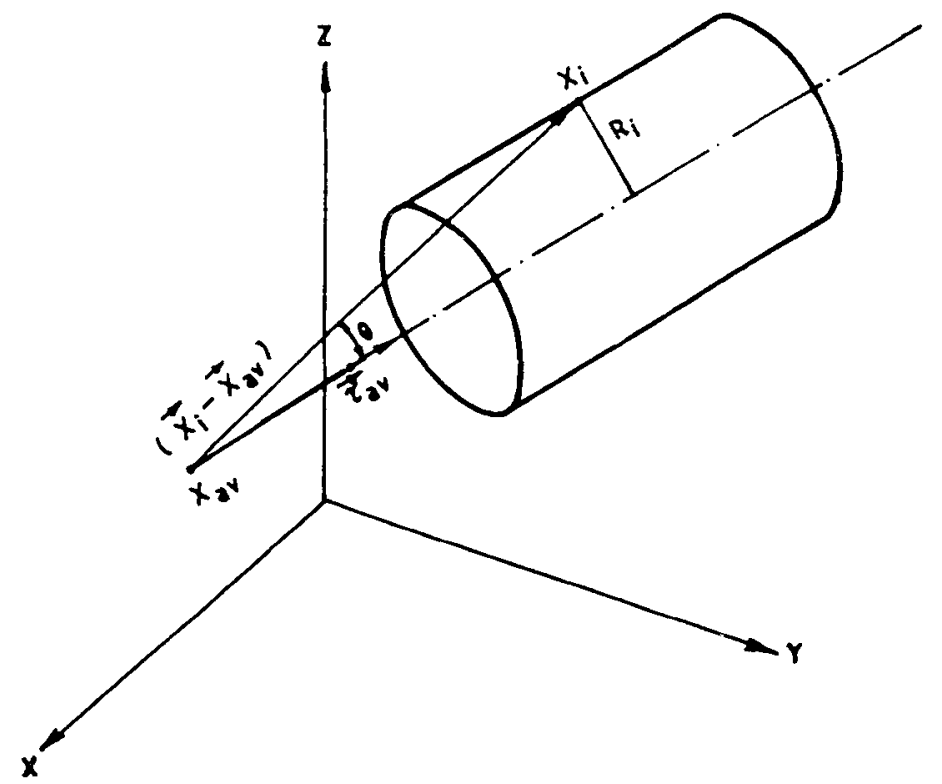

Figure 4. Estimation of the radius of a cylinder. 
However in practice, such a unique point cannot be determined due to the same reasons as stated earlier. Hence, the apex is estimated in the following manner. For all points $\mathbf{X}_{i}$ with normals $\mathbf{N}_{i}$, the equation of the plane, tangent to the surface at $\mathbf{X}_{i}$ is obtained. Taking the intersection of three such pairwise nonparallel planes (Shanti Narayan 1985) an estimate $V_{k}$ of the apex is obtained. Average of all such estimates gives the mean apex position $\mathbf{V}_{\mathrm{av}}$. The uncertainty in the estimation of the position of apex is given by the standard deviation $\sigma_{v}$ of apex.

Once the position of the apex $\left(\mathbf{V}_{\mathrm{av}}\right)$ is fixed, the axis of the conical surface is estimated in the following manner. $\forall i, 1 \leqslant i \leqslant M$, the normal $h_{i}$ to the plane containing the points $\mathbf{V}_{\text {av }}, \mathbf{X}_{i}$ and $\mathbf{N}_{i}$ (normal to the surface at $\mathbf{X}_{i}$ ) is determined as follows (refer figure 5).

$$
\mathbf{h}_{i}=\left(\mathbf{X}_{i}-\mathbf{V}_{\mathrm{av}}\right) \times \mathbf{N}_{\boldsymbol{i}} \text {. }
$$

The orientation of the axis of the cone is given by the cross product of two such nonparallel vectors $\mathbf{h}_{i}$ and $\mathbf{h}_{j}$, where $1 \leqslant i, j \leqslant M$ and $i \neq j$. Thus $\forall i, j$, where $\mathbf{h}_{i}$ and $\mathbf{h}_{j}$ are not parallel, the $k$ th estimate of the axis orientation is given by the vector $\tau_{k}$ where

$$
\tau_{k}=\left(\mathbf{h}_{i} \times \mathbf{h}_{j}\right) /\left|\mathbf{h}_{i} \times \mathbf{h}_{j}\right|,
$$

From these estimates the expected orientation $\tau_{\mathrm{av}}$ and the standard deviation $\sigma_{\tau}$ are computed.

Once the apex $V_{\mathrm{av}}$ and the axis orientation are available, for each point $X_{i}$ an estimate of the semi apex angle $\gamma_{i}$ is obtained

$$
\sin \gamma_{i}=\left|\left(\mathbf{X}_{i}-\mathbf{V}_{\mathrm{av}}\right) \times \tau_{\mathrm{av}}\right| / /\left(\mathbf{X}_{i}-\mathbf{X}_{\mathrm{av}}\right) \mid
$$

From different estimates of $\gamma$ we can compute average semi apex angle $\gamma_{\mathrm{av}}$ and standard deviation $\sigma_{\gamma}$. The standard deviations of the apex, the axis and the semi apex angle together give a measure of uncertainty in fitting the conical surface to the set of points.

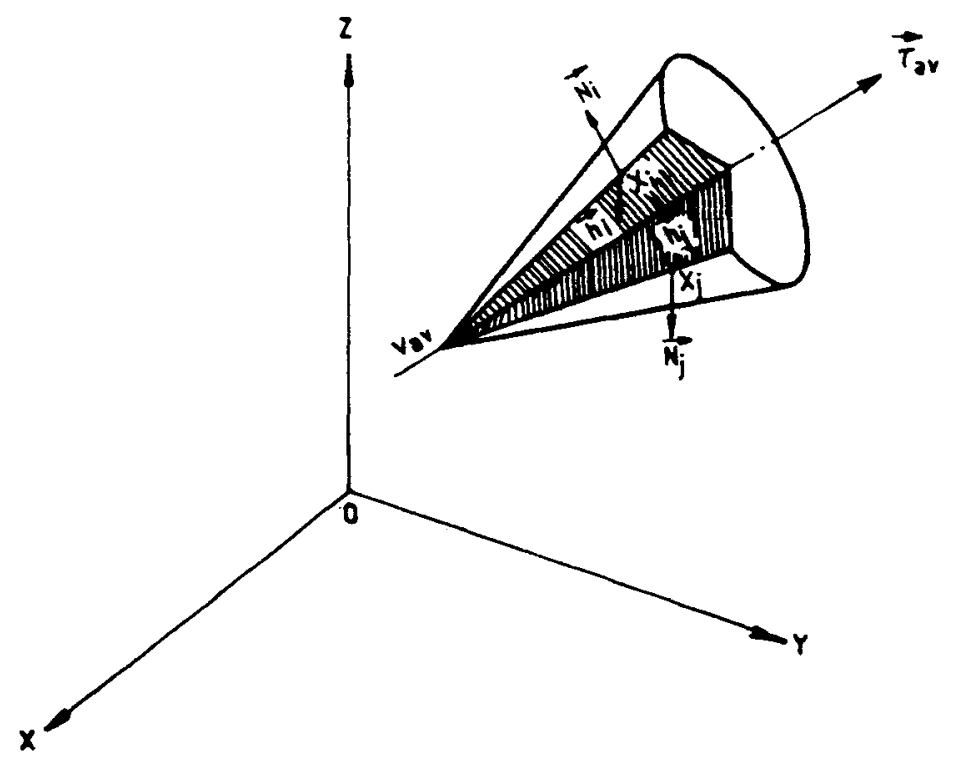

Figure.5. Estimation of the axis of a cone. 


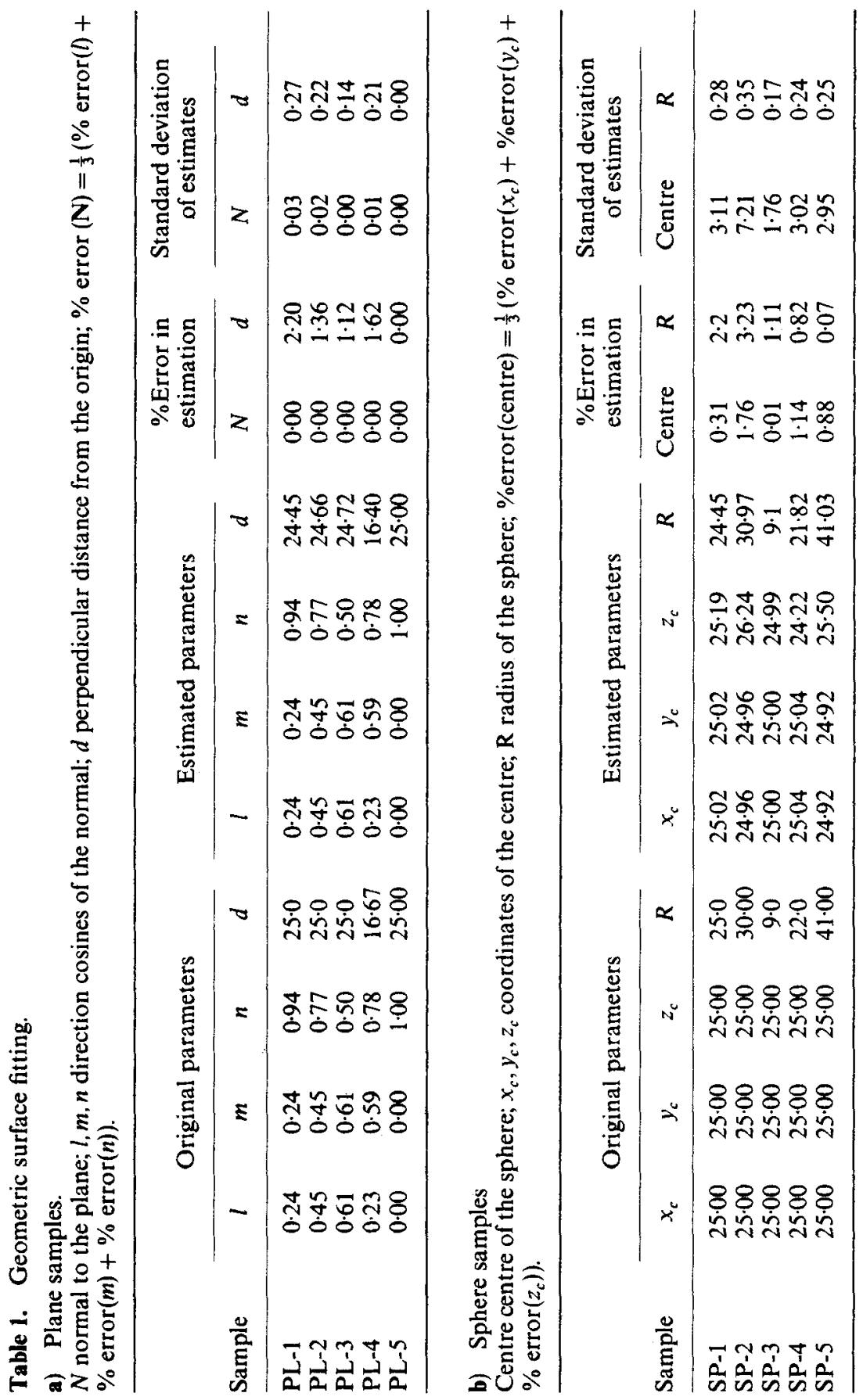



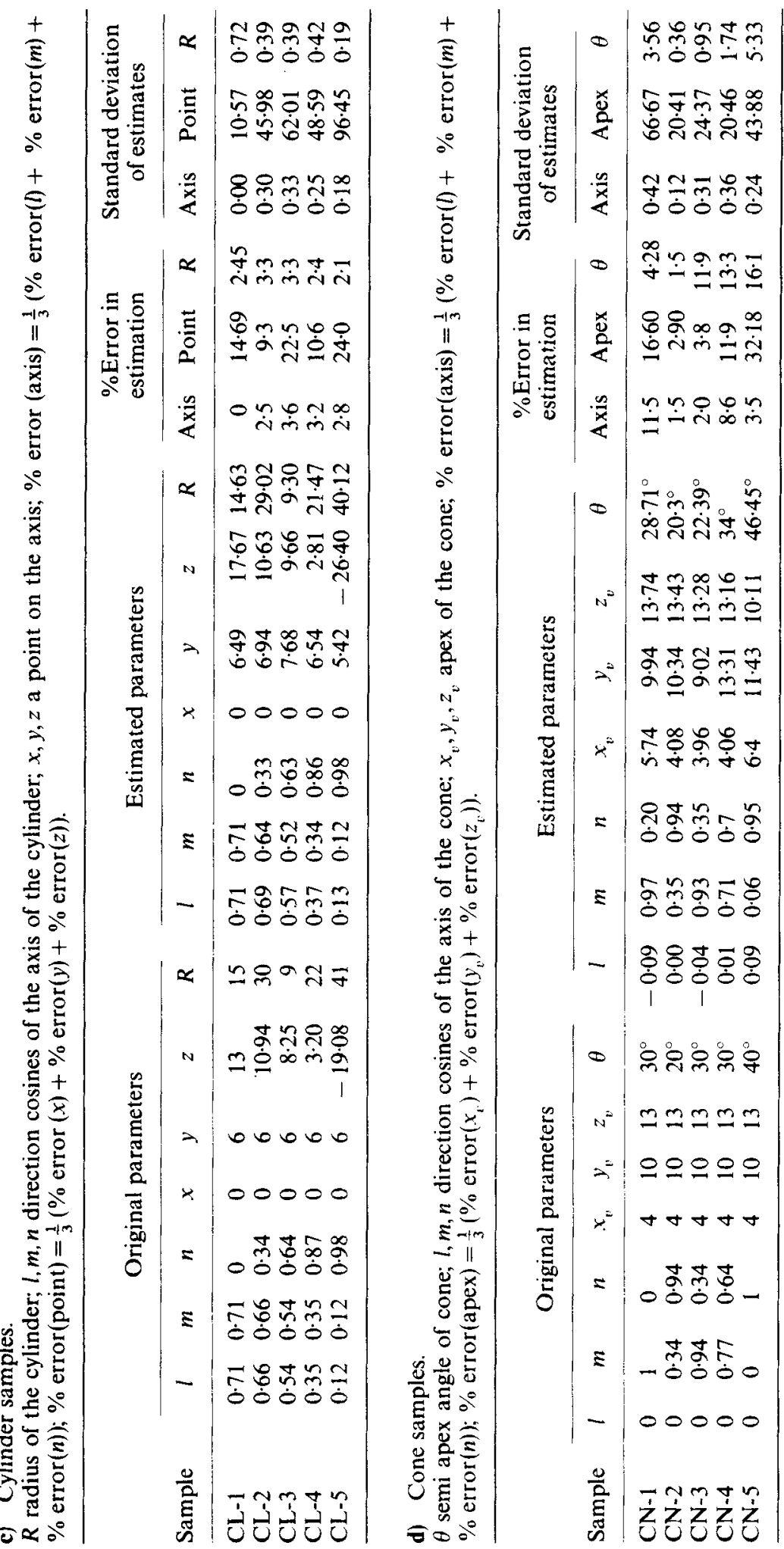


\subsection{Experimental results}

Experimental results on synthetic range images of size $50 \times 50$ are presented. To reduce the amount of computation, surface normals are computed at some sample points, distributed uniformly over the surface patch. At each sample point, surface normal is estimated by fitting a plane to a $5 \times 5$ neighbourhood of the point, using least square error method. A set of five images from each of the classes plane, sphere, cylinder and cone are considered. For each sample the corresponding surface is fitted geometrically. The results obtained are tabulated in table 1 .

It is observed from table 1 that the percentage of errors in the estimated parameter values are quite low for plane and sphere fitting $(<3.5 \%)$. For the cylinder samples the percentage errors in the estimations of axis orientation are low $(<3.6 \%)$ and also in the estimations of radius $(<3.3 \%)$. Estimation of a point on the axis has given a large error in most cases $(10 \cdot 6-24 \%)$. This is due to the fact that even a slight error in estimating the axis orientation can significantly change the estimated point position. In the case of cone samples, the error in estimation of axis orientation is quite low $(1.5-11.5 \%)$. Angle estimation also has low percentage error $(1 \cdot 5-16 \cdot 1 \%)$. The error in estimation of apex position has shown wide variation $(2 \cdot 9 \%$ to $32 \cdot 2 \%)$. From the results on synthetic range images it can be said that errors in an orientation estimation (e.g., axis, normal) or a distance estimation (e.g., radius) have been less as compared to estimation of a point position (e.g., apex, centre).

\section{Fuzzy characterization of surfaces}

In the previous section on geometric surface fitting it has been shown how an appropriate surface may be fitted when the input image is of planar, spherical, cylindrical or conical types. The implicit assumption has been that a priori information regarding the surface nature is available. But even in the absence of $a$ priori information the geometric surface fitting proves to be useful. In such cases all four surfaces are fitted to the set of points and in each case the uncertainties, as given by the standard deviations of the parameters, are noted. These measures of uncertainty in parameter estimation are used to classify the surface as a planar or spherical or cylindrical or conical surface. The details of the method involved are discussed in this section.

The simplest strategy to design the classifier is to assign the input image to that class whose parameters have been estimated with the least amount of uncertainty. However, the solution is not so straightforward because parameters for different types of surfaces are obtained with different methods of estimation. Also the numbers of parameters for different types of surfaces are different. This has led to the development of a fuzzy classifier, where two thresholds are set for the standard deviation of each parameter of a particular surface. The lower threshold denotes the maximum uncertainty expected when the surface patch in the input image is of the same type as the surface fitted. The upper threshold denotes the least amount of uncertainty expected when the input is from any class other than that of the surface fitted. The input is assigned a membership to the class by comparing the standard deviation values obtained for a parameter of the fitted surface with the corresponding thresholds. Fuzziness in the classification is introduced by grading the membership if a standard deviation value falls between the two thresholds. Finally, the membership values obtained with each parameter of a fitted surface type are 
combined to get an overall membership. This procedure is repeated for each of the four surface types namely, planar, spherical, cylindrical and conical surfaces. The result is a membership vector giving the membership of the input surface with respect to the four different classes. The vector itself can be taken as the characterization of the surface. Alternatively, the sample is classified into the class with the maximum membership value (the dominant class) and characterized by the corresponding parameters. Designing the classifier involves finding the thresholds, the details of which are discussed below.

\subsection{Classifier design}

A set of images, henceforth referred to as training images or samples, are taken from each of the four classes. All four surfaces are fitted geometrically to each of the samples. In each case the parameters and their standard deviations are noted. The fact that for any particular type of fitted surface, samples from the same class should yield lower standard deviation values as compared to samples from other classes, is used to obtain the lower thresholds. If $\theta$ is a parameter of a class denoted by class $(\theta)$, then the lower threshold for $\theta$ is defined as

where

$$
l o_{\theta}=\text { mean }\left(\sigma_{\theta_{i}}\right)+\text { standard deviation }\left(\sigma_{\theta_{i}}\right),
$$

$$
\begin{aligned}
i & =1 . . M \text { is the set of sample images from class }(\theta), \\
\theta_{i} & =\text { value of parameter } \theta \text { for the } i \text { th sample, } \\
\sigma_{\theta_{i}} & =\text { standard deviation in estimating } \theta_{i} .
\end{aligned}
$$

The upper threshold for parameter $\theta$ can be set by considering the standard deviations obtained with sample images from all classes other than class $(\theta)$. Thus the upper threshold is defined as

$$
h i_{\theta}=\min \left(\sigma_{\theta_{k}}\right) \text {, }
$$

for all $k$, where $k=1 . . N$ is the set of samples not belonging to class $(\theta)$ and $\sigma_{\theta_{k}}>l o_{\theta}$.

The value of $l o_{\theta}$ has been selected considering the distribution of standard deviation values of the samples from the same class as that of the surface fitted. The value of $h i_{\theta}$ has been selected in order to reduce the misclassification of samples from other classes into the class of the surface fitted.

The membership function for $\operatorname{class}(\theta)$ with respect to the parameter $\theta$ can be defined as $\mu_{\theta}$ where

$$
\begin{array}{ll}
\mu_{\theta}=1, & \text { if } \sigma_{\theta} \leqslant l o_{\theta}, \\
\mu_{0}=\left(h i_{\theta}-\sigma_{\theta}\right) /\left(h i_{\theta}-l o_{\theta}\right), & \text { if } l o_{\theta} \leqslant \sigma_{\theta} \leqslant h i_{\theta}, \\
\mu_{\theta}=0, & \text { if } h i_{\theta} \leqslant \sigma_{\theta} .
\end{array}
$$

The membership function $\mu_{\theta}$ is graphically shown in figure 6 .

Overall membership of a sample in a class can now be defined in terms of the membership with respect to individual parameters of the class. For a class $\omega$ if $\theta^{d}, d=1 \ldots D_{\omega}$ are the parameters which characterize the class, the overall class membership $\mu_{\omega}$ for a sample is given by

$$
\mu_{\omega}=\left(1 / D_{\omega}\right) \sum_{d=1}^{D_{c}} \mu_{\theta^{\star}},
$$




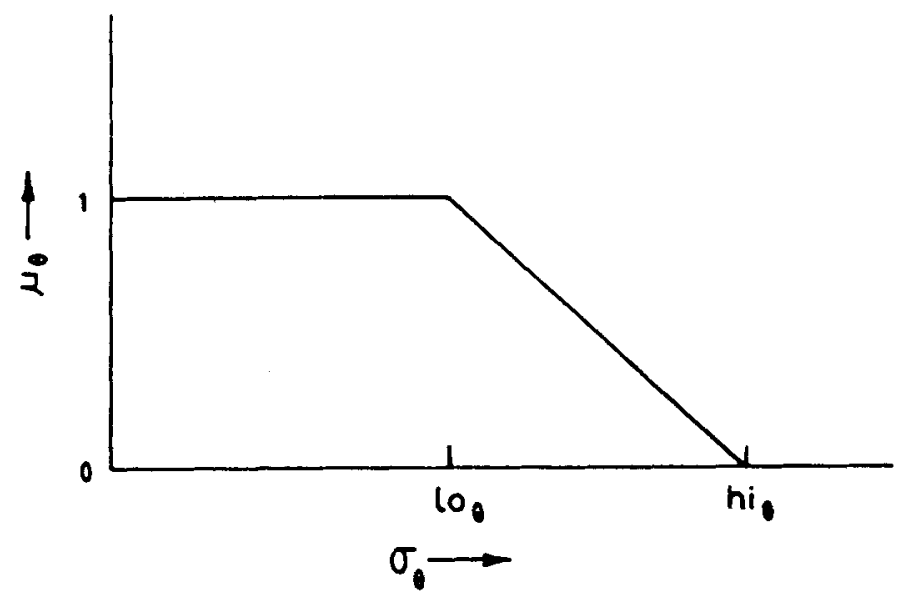

Figure 6. Fuzzy membership function $\left(\mu_{\theta}\right)$.

where the class $\omega$ represents one of \{plane, sphere, cylinder, cone $\}$ and $\mu_{\theta^{a}}$ is the membership obtained for parameter $\theta^{d}$ as per (13).

\subsection{Experimental results and discussions}

To design the classifier five training samples from each of the four classes are taken. The standard deviations of different parameters obtained when all the four surfaces are fitted to each training sample are listed in table 2. The lower and upper thresholds for each parameter are tabulated in table 3 .

Once the classifier is designed, classification results for other images from the four classes (henceforth referred to as test samples) are presented. For each test sample all the four surfaces were fitted and the standard deviations of the various parameters were noted. From the standard deviations, the membership values with respect to the different classes were derived using the corresponding membership functions ((30) and (31)). The results are tabulated in table 4 . The table also shows the dominant class displayed by each of these test samples. It may be seen that only one sample out of 20 has been misclassified. Another point of interest is the nature of the membership vectors for test samples from various classes. Some planar test samples have shown conical behaviour as well (although not dominant). When a cone is to be fitted to a surface, each apex estimate is formed from three tangential planes where the line of intersection of two planes is not parallel to the third. Ideally, for a planar surface, all surface normals are parallel. Hence, fitting a cone fails at the stage of finding the apex itself, since no suitable combinations of three pairwise nonparallel tangential planes can be found. This conical behaviour is seen only in cases where the effects of quantization noise in the input image are so severe that it has been possible to find combinations of three normals to find apex estimates. In all these cases the semi apex angle is close to $90^{\circ}$ and axis of the cone is normal to the plane. Thus conical behaviour exhibited by the planar samples is acceptable since any plane can be considered as a cone with semi apex angle of $90^{\circ}$. Sphere samples also have exhibited conical behaviour. Sphere samples actually represent the visible portion of a sphere which may be a hemisphere or a part of it. In all 
Table 2. Standard deviations of the parameters obtained when all the four surface types are fitted to the training samples.

a) Planar training samples

Unable to fit surface; plane: $\sigma_{N}$ s.d. of the normal direction, $\sigma_{d}$ s.d. of perpendicular distance from origin; sphere: $\sigma_{X}$ s.d. of the centre, $\sigma_{R}$ s.d. of the radius; cylinder: $\sigma_{z}$ s.d. of axis orientation, $\sigma_{X}$ s.d. of a point on the axis, $\sigma_{R}$ s.d. of the radius; cone: $\sigma_{v}$ s.d. of the apex, $\sigma_{\tau}$ s.d. of the axis orientation. $\sigma_{\theta}$ s.d. of the semi apex angle.

\begin{tabular}{|c|c|c|c|c|c|c|c|c|c|c|}
\hline \multirow[b]{3}{*}{ Sample } & \multicolumn{10}{|c|}{ Type of surface fitted } \\
\hline & \multicolumn{2}{|c|}{ Plane } & \multicolumn{2}{|c|}{ Sphere } & \multicolumn{3}{|c|}{ Cylinder } & \multicolumn{3}{|c|}{ Cone } \\
\hline & $\sigma_{N}$ & $\sigma_{d}$ & $\sigma_{x}$ & $\sigma_{R}$ & $\sigma_{\tau}$ & $\sigma_{x}$ & $\sigma_{R}$ & $\sigma_{v}$ & $\sigma_{\tau}$ & $\sigma_{\theta}$ \\
\hline PL-1 & $0 \cdot 00$ & 0.00 & - & - & - & - & - & - & - & - \\
\hline PL-2 & $0 \cdot 02$ & 0.27 & $406 \cdot 42$ & 6.98 & 0.54 & $1248 \cdot 51$ & $8 \cdot 32$ & - & 一 & - \\
\hline PL-3 & $0-01$ & 0.22 & - & - & 0.40 & $642 \cdot 61$ & 3.74 & - & - & - \\
\hline PL-4 & 0.00 & 0.14 & - & - & 0.44 & 826.92 & 4.62 & - & 一 & - \\
\hline PL-5 & $0-01$ & 0.22 & - & - & 0.81 & $653 \cdot 74$ & 3.89 & $81 \cdot 42$ & 0.98 & 0.92 \\
\hline
\end{tabular}

b) Spherical training samples.

Unabie to fit surface; plane: $\sigma_{N}$ s.d. of the normal direction, $\sigma_{d}$ s.d. of perpendicular distance from origin; sphere: $\sigma_{X}$ s.d. of the centre, $\sigma_{R}$ s.d. of the radius; cylinder: $\sigma_{\tau}$ s.d. of axis orientation, $\sigma_{X}$ s.d. of a point on the axis, $\sigma_{R}$ s.d. of the radius; cone: $\sigma_{V}$ s.d. of the apex, $\sigma_{\tau}$ s.d. of the axis orientation, $\sigma_{\theta}$ s.d. of the semi apex angle.

Type of surface fitted

\begin{tabular}{|c|c|c|c|c|c|c|c|c|c|c|}
\hline \multirow[b]{2}{*}{ Sample } & \multicolumn{2}{|c|}{ Plane } & \multicolumn{2}{|c|}{ Sphere } & \multicolumn{3}{|c|}{ Cylinder } & \multicolumn{3}{|c|}{ Cone } \\
\hline & $\sigma_{N}$ & $\sigma_{d}$ & $\sigma_{x}$ & $\sigma_{R}$ & $\sigma_{\mathrm{r}}$ & $\sigma_{x}$ & $\sigma_{R}$ & $\sigma_{V}$ & $\sigma_{t}$ & $\sigma_{\theta}$ \\
\hline SP-1 & 0.74 & 3.78 & $3 \cdot 11$ & 0.28 & 0.84 & $494 \cdot 2$ & $2 \cdot 27$ & $64 \cdot 87$ & 0.72 & 0.41 \\
\hline$S P-2$ & 0.67 & 5.77 & 7.21 & 0.35 & 0.81 & $227 \cdot 1$ & 3.38 & $126 \cdot 77$ & 0.78 & 0.73 \\
\hline SP-3 & 0.78 & 2.89 & 1.76 & $0 \cdot 17$ & 0.85 & 197.8 & 1.90 & 98.39 & 0.76 & 0.68 \\
\hline SP-4 & 0.73 & 4.77 & 3.02 & $0 \cdot 20$ & 0.83 & $522 \cdot 2$ & 3.08 & $95 \cdot 25$ & 0.74 & 0.4 \\
\hline SP-5 & 0.49 & 3.42 & 2.95 & 0.25 & 0.79 & 301.0 & $2 \cdot 40$ & $86 \cdot 12$ & 0.97 & 0.44 \\
\hline
\end{tabular}

c) Cylinder training samples.

Unable to fit surface; plane: $\sigma_{N}$ s.d. of the normal direction, $\sigma_{d}$ s.d. of perpendicular distance from origin; sphere: $\sigma_{X}$ s.d. of the centre, $\sigma_{R}$ s.d. of the radius; cylinder: $\sigma_{\tau}$ s.d. of axis orientation, $\sigma_{X}$ s.d. of a point on the axis, $\sigma_{R}$ s.d. of the radius; cone: $\sigma_{V}$ s.d. of the apex, $\sigma_{r}$ s.d. of the axis orientation. $\sigma_{\theta}$ s.d. of the semi apex angle.

Type of surface fitted

\begin{tabular}{|c|c|c|c|c|c|c|c|c|c|c|}
\hline \multirow[b]{2}{*}{ Sample } & \multicolumn{2}{|c|}{ Plane } & \multicolumn{2}{|c|}{ Sphere } & \multicolumn{3}{|c|}{ Cylinder } & \multicolumn{3}{|c|}{ Cone } \\
\hline & $\sigma_{N}$ & $\sigma_{d}$ & $\sigma_{x}$ & $\sigma_{R}$ & $\sigma_{\mathrm{t}}$ & $\sigma_{x}$ & $\sigma_{R}$ & $\sigma_{V}$ & $\sigma_{\tau}$ & $\sigma_{\theta}$ \\
\hline CL-1 & 0.57 & $3 \cdot 27$ & $19 \cdot 20$ & $5 \cdot 14$ & 0.00 & $10 \cdot 57$ & 0.72 & - & 一 & - \\
\hline CL-2 & 0.44 & 3.97 & $39 \cdot 51$ & 3.56 & $0 \cdot 30$ & 45.98 & 0.30 & 295.45 & 0.46 & 0.98 \\
\hline CL-3 & 0.59 & $2 \cdot 16$ & 56.66 & $9 \cdot 02$ & 0.33 & 62.00 & 0.58 & $129 \cdot 59$ & 0.80 & $0 \cdot 37$ \\
\hline CL-4 & 0.57 & 5.01 & 55.09 & $12 \cdot 37$ & 0.25 & $48 \cdot 59$ & 0.41 & 463.24 & 0.77 & 0.30 \\
\hline CL-5 & 0.36 & $3 \cdot 57$ & 85.91 & 39.63 & 0.18 & 96.45 & 0.19 & $629 \cdot 80$ & 0.82 & 0.38 \\
\hline
\end{tabular}


Table 2. (Continued)

d) Conical training samples.

Unable to fit surface; plane: $\sigma_{N}$ s.d. of the normal direction, $\sigma_{d}$ s.d. of perpendicular distance from origin; sphere: $\sigma_{X}$ s.d. of the centre, $\sigma_{R}$ s.d. of the radius; cylinder: $\sigma_{\tau}$ s.d. of axis orientation, $\sigma_{X}$ s.d. of a point on the axis, $\sigma_{R}$ s.d. of the radius; cone: $\sigma_{V}$ s.d. of the apex, $\sigma_{\tau}$ s.d. of the axis orientation, $\sigma_{\theta}$ s.d. of the semi apex angle.

\begin{tabular}{|c|c|c|c|c|c|c|c|c|c|c|}
\hline \multirow[b]{3}{*}{ Sample } & \multicolumn{10}{|c|}{ Type of surface fitted } \\
\hline & \multicolumn{2}{|c|}{ Plane } & \multicolumn{2}{|c|}{ Sphere } & \multicolumn{3}{|c|}{ Cylinder } & \multicolumn{3}{|c|}{ Cone } \\
\hline & $\sigma_{N}$ & $\sigma_{d}$ & $\sigma_{x}$ & $\sigma_{R}$ & $\sigma_{\tau}$ & $\sigma_{x}$ & $\sigma_{R}$ & $\sigma_{v}$ & $\sigma_{\tau}$ & $\sigma_{\theta}$ \\
\hline $\mathrm{CN}-1$ & 0.30 & $9 \cdot 5$ & $66 \cdot 42$ & $3 \cdot 15$ & 0.64 & $158 \cdot 34$ & $1 \cdot 24$ & 66.67 & 0.42 & 0.06 \\
\hline $\mathrm{CN}-2$ & 0.74 & $3 \cdot 1$ & $45 \cdot 31$ & $94 \cdot 11$ & 0.44 & $164 \cdot 12$ & 21.73 & $20 \cdot 41$ & 0.12 & 0.01 \\
\hline $\mathrm{CN}-3$ & 0.43 & $2 \cdot 15$ & $32 \cdot 8$ & 18.84 & 0.73 & 330.0 & $4 \cdot 21$ & $24 \cdot 37$ & 0.31 & 0.02 \\
\hline $\mathrm{CN}-4$ & 0.50 & $3 \cdot 29$ & $35 \cdot 30$ & $2 \cdot 86$ & 0.63 & 784.8 & 7.93 & 20.46 & 0.36 & 0.03 \\
\hline CN.5 & 0.48 & 3.64 & 47.79 & 4.57 & 0.74 & $105 \cdot 29$ & 2.47 & 43.88 & 0.24 & 0.09 \\
\hline
\end{tabular}

these cases, the axis of the fitted cone is perpendicular to the plane of the image, i.e., along the $z$-axis. The standard deviations of the apex and the axis are low enough to fall below the corresponding lower threshold. These two parameters thus contribute a membership value of 1 each. The third parameter, the apex angle, has given a high standard deviation above the higher threshold. Thus contribution of this parameter to overall membership function is 0 . Thus an overall membership of 0.67 has resulted for these samples when a cone is fitted. The 0.33 membership with respect to cylindrical classes shown by sphere samples can be explained similarly. The point estimate has given a low standard deviation below the lower threshold in all the cases while the other two parameters have crossed the higher threshold. Cylinder samples have shown no other behaviour significantly apart from cylindrical behaviour. Two cone samples have shown some amount of planar behaviour out of which one cone sample has been misclassified as planar because in that case value of the semiapex angle was very high.

Table 3. Lower and upper thresholds of different parameters for different surface classes

$\mathrm{PL}=$ planar surface; $\mathrm{SP}=$ spherical surface; $\mathrm{CL}=$ cylindrical surface; $\mathrm{CN}=$ conical surface.

\begin{tabular}{llcc}
\hline $\begin{array}{l}\text { Surface } \\
\text { type }\end{array}$ & Parameters & $\begin{array}{c}\text { Lower } \\
\text { threshold }\end{array}$ & $\begin{array}{c}\text { Upper } \\
\text { threshold }\end{array}$ \\
\hline PL & Normal $N$ & 0.015 & 0.30 \\
& Distance $d$ & 0.28 & $2 \cdot 15$ \\
SP & Centre $X c$ & $5 \cdot 48$ & $19 \cdot 2$ \\
& Radius $R$ & 0.31 & $2 \cdot 86$ \\
& Axis $\tau$ & 0.33 & $0 \cdot 40$ \\
CL & Point $X$ & 80.42 & $105 \cdot 29$ \\
& Radius $R$ & 0.63 & $1 \cdot 24$ \\
& Apex $V$ & $53 \cdot 15$ & 64.87 \\
CN & Axis $\tau$ & 0.39 & 0.46 \\
& Angle $\theta$ & 0.07 & 0.30 \\
\hline
\end{tabular}


Table 4. Fuzzy classification of plane, sphere, cylinder and cone samples.

\begin{tabular}{lcccccc}
\hline \multicolumn{5}{c}{ Membership vector } & & \\
Sample & PL & SP & CL & CN & $\begin{array}{c}\text { Dominant } \\
\text { class }\end{array}$ & $\begin{array}{c}\text { Correctly classified } \\
(Y / N)\end{array}$ \\
\hline PL-6 & 0.93 & 0.06 & 0.00 & 0.67 & PL & Y \\
PL-7 & 1.00 & 0.00 & 0.00 & 0.67 & PL & Y \\
PL-8 & 0.98 & 0.38 & 0.00 & 0.88 & PL & Y \\
PL-9 & 0.94 & 0.00 & 0.00 & 0.67 & PL & Y \\
PL-10 & 1.00 & 0.00 & 0.00 & 0.00 & PL & Y \\
SP-6 & 0.00 & 1.00 & 0.33 & 0.67 & SP & Y \\
SP-7 & 0.00 & 1.00 & 0.33 & 0.67 & SP & Y \\
SP-8 & 0.00 & 1.00 & 0.33 & 0.67 & SP & Y \\
SP-9 & 0.00 & 1.00 & 0.33 & 0.67 & SP & Y \\
SP-10 & 0.10 & 1.00 & 0.33 & 0.67 & SP & Y \\
CL-6 & 0.00 & 0.00 & 1.00 & 0.00 & CL & Y \\
CL-7 & 0.00 & 0.00 & 1.00 & 0.00 & CL & Y \\
CL-8 & 0.07 & 0.00 & 1.00 & 0.30 & CL & Y \\
CL-9 & 0.00 & 0.00 & 1.00 & 0.00 & CL & Y \\
CL-10 & 0.00 & 0.00 & 0.54 & 0.22 & CL & Y \\
CN-6 & 0.00 & 0.00 & 0.03 & 0.33 & CN & Y \\
CN-7 & 0.00 & 0.00 & 0.00 & 0.52 & CN & Y \\
CN-8 & 0.00 & 0.09 & 0.00 & 0.41 & CN & Y \\
CN-9 & 0.53 & 0.00 & 0.00 & 1.00 & CN & Y \\
CN-10 & 0.59 & 0.00 & 0.00 & 0.22 & PL & N \\
\hline A & &
\end{tabular}

Abbreviations as in table 3

Classification results for general quadratic surfaces not belonging to any of the four classes, plane, sphere, cylinder and cone, are also presented. The aim is to see if a general pattern of membership vectors is derivable for a set of samples from the same quadratic class. This may be useful in characterising that quadratic class. The results of this experimentation are given in table 5 . The classification based on the dominant class behaviour is also indicated in the table. It is interesting to note that samples from the same quadratic class show the same dominant class behaviour. For example cylindrical behaviour is predominantly shown by samples from elliptical and hyperbolic cylinder classes, while ellipsoids are predominantly spherical, hyperboloids are predominantly conical.

\section{Occluded surface characterization}

In a range image with more than one object, usually some objects may be partially occluded by the others. This makes the problem of understanding range images more difficult since certain regions in the image representing surfaces of objects may be missing or partially occluded. Since due to occlusion the object surfaces are partially visible, local surface properties should be used to characterize them. The geometric method for surface characterization, discussed in $\$ 2$, obtains surface descriptions through surface normal analysis. Since the normal to a surface at a point is the local property of the surface at the point with respect to the point's neighbourhood, 
Table 5. Fuzzy classification of general quadric surface samples.

\begin{tabular}{|c|c|c|c|c|c|c|}
\hline \multirow[b]{2}{*}{ Surface type } & \multirow[b]{2}{*}{ Sample } & \multicolumn{4}{|c|}{ Membership vector } & \multirow{2}{*}{$\begin{array}{l}\text { Dominan } \\
\text { class }\end{array}$} \\
\hline & & PL & $\mathrm{SP}$ & $\mathrm{CL}$ & $\mathrm{CN}$ & \\
\hline $\begin{array}{l}\text { Elliptic } \\
\text { cylinder }\end{array}$ & $\begin{array}{l}1 \\
2 \\
3 \\
4 \\
5\end{array}$ & $\begin{array}{l}0.00 \\
0.40 \\
0.00 \\
0.43 \\
0.00\end{array}$ & $\begin{array}{l}0.19 \\
0.00 \\
0.19 \\
0.15 \\
0.00\end{array}$ & $\begin{array}{l}0.67 \\
1.00 \\
0.67 \\
1.00 \\
0.33\end{array}$ & $\begin{array}{l}0.00 \\
0.33 \\
0.00 \\
0.00 \\
0.00\end{array}$ & $\begin{array}{l}C L \\
C L \\
C L \\
C L \\
C L\end{array}$ \\
\hline $\begin{array}{l}\text { Hyperbolic } \\
\text { cylinder }\end{array}$ & $\begin{array}{l}1 \\
2 \\
3 \\
4 \\
5\end{array}$ & $\begin{array}{l}0.00 \\
0.07 \\
0.00 \\
0.00 \\
0.00\end{array}$ & $\begin{array}{l}0.00 \\
0.28 \\
0.00 \\
0.09 \\
0.00\end{array}$ & $\begin{array}{l}0.33 \\
1.00 \\
0.67 \\
0.78 \\
0.33\end{array}$ & $\begin{array}{l}0.00 \\
0.00 \\
0.00 \\
0.00 \\
0.00\end{array}$ & $\begin{array}{l}\text { CL } \\
C L \\
C L \\
C L \\
C L\end{array}$ \\
\hline Ellipsoid & $\begin{array}{l}1 \\
2 \\
3 \\
4 \\
5\end{array}$ & $\begin{array}{l}0 \cdot 00 \\
0 \cdot 00 \\
0 \cdot 11 \\
0 \cdot 16 \\
0 \cdot 22\end{array}$ & $\begin{array}{l}0.40 \\
0.53 \\
0.93 \\
0.93 \\
0.99\end{array}$ & $\begin{array}{l}0.33 \\
0.33 \\
0 \cdot 33 \\
0.33 \\
0.46\end{array}$ & $\begin{array}{l}0.33 \\
0.25 \\
0.33 \\
0.36 \\
0.67\end{array}$ & $\begin{array}{l}\text { SP } \\
\text { SP } \\
\text { SP } \\
\text { SP } \\
\text { SP }\end{array}$ \\
\hline $\begin{array}{l}\text { Elliptic } \\
\text { cone }\end{array}$ & $\begin{array}{l}1 \\
2 \\
3 \\
4 \\
5\end{array}$ & $\begin{array}{l}0.00 \\
0.27 \\
0.00 \\
0.00 \\
0.00\end{array}$ & $\begin{array}{l}0.00 \\
0.55 \\
0.00 \\
0.00 \\
0.00\end{array}$ & $\begin{array}{l}0.00 \\
0.00 \\
0 \cdot 11 \\
0.00 \\
0.00\end{array}$ & $\begin{array}{l}1.00 \\
1.00 \\
1.00 \\
0.67 \\
0.33\end{array}$ & $\begin{array}{l}C N \\
C N \\
C N \\
C N \\
C N\end{array}$ \\
\hline Hyperboloid & $\begin{array}{l}1 \\
2 \\
3 \\
4 \\
5\end{array}$ & $\begin{array}{l}0.00 \\
0.00 \\
0.00 \\
0.00 \\
0.00\end{array}$ & $\begin{array}{l}0.00 \\
0.00 \\
0.00 \\
0.00 \\
0.00\end{array}$ & $\begin{array}{l}0.00 \\
0.00 \\
0.00 \\
0.31 \\
0.00\end{array}$ & $\begin{array}{l}0.33 \\
0.33 \\
0.33 \\
0.33 \\
0.33\end{array}$ & $\begin{array}{l}\text { CN } \\
C N \\
C N \\
C N \\
\text { CN }\end{array}$ \\
\hline $\begin{array}{l}\text { Hyperbolic } \\
\text { paraboloid }\end{array}$ & $\begin{array}{l}1 \\
2 \\
3 \\
4 \\
5\end{array}$ & $\begin{array}{l}0.00 \\
0.00 \\
0.00 \\
0.00 \\
0.00\end{array}$ & $\begin{array}{l}0.00 \\
0.00 \\
0.00 \\
0.00 \\
0.00\end{array}$ & $\begin{array}{l}0.00 \\
0.00 \\
0.00 \\
0.00 \\
0.00\end{array}$ & $\begin{array}{l}0.33 \\
0.27 \\
0.33 \\
0.33 \\
0.33\end{array}$ & $\begin{array}{l}\mathrm{CN} \\
\mathrm{CN} \\
\mathrm{CN} \\
\mathrm{CN} \\
\mathrm{CN}\end{array}$ \\
\hline
\end{tabular}

Abbreviations as in table 3

the geometric method also forms a reliable tool for characterizing partially visible surfaces.

The shape of a surface (i.e., planar, spherical, cylindrical or conical) is uniquely determined by the global parameters of the surface. For example, a spherical surface is uniquely characterized by its centre and radius. The geometric method estimates the global surface parameters from the locally calculated surface normals. Since the estimation of global parameters at different regions of a surface should be very close to each other, it is also possible to determine if two or more partially visible surface patches are parts of the same surface. This is achieved in the following manner.

Let a surface class $\omega, \omega \in$ \{plane, sphere, cylindrical, cone\}, be characterized by a set of parameters $\left\{\theta^{d} \mid 1 \leqslant d \leqslant D_{\omega}\right\}$, where $D_{\omega}$ is constant whose value is different for different surface classes. This set of parameters defines a $D_{\omega}$-dimensional space, henceforth referred to as parameter space, such that the mean values of the parameters $\theta_{i}^{d}$ describing a surface $S_{i}$ of class $\omega$ represent a point $p_{i}$ in the parameter space. Because 
of the uncertainty in the measurement of $\theta_{i}^{d}$, different estimates of $\theta_{i}^{d}$ may occur anywhere in the region $\left(\theta_{i}^{\mathrm{d}}-h i_{\theta_{i}^{d}}\right)$ to $\left(\theta_{i}^{d}+h i_{\delta_{i}^{f}}\right)$ where $h i_{\theta_{i}^{d}}$ is the upper threshold used in the fuzzy classifier and is given in (29). Thus different estimates of the point $p_{i}$ may occur anywhere within a hyper rectangular parallelopiped bounded within the region $\left[\theta_{i}^{d}-h i_{\sigma_{i}}, \theta_{i}^{d}+h i_{\sigma_{i}}\right]$ for $1 \leqslant d \leqslant D_{\omega}$. This region is referred to as region of occurrence of $S_{i}$. Let this region for surface $S_{i}$ be denoted by $R_{i}$. Now two partially visible surface patches $S_{i}$ and $S_{j}$, both of same surface type $\omega$, are assumed to be possible parts of the same surface if $R_{i} \cap R_{j} \neq \phi$. If this condition is satisfied then surfaces are fitted over the region $S_{i} \cup S_{j}$. If the fitted surface also shows a dominant class $\omega$ then $S_{i}$ and $S_{j}$ are identified as parts of the same surface and the parameters of the fitted surface are extracted. If the fitted surface shows a dominant class other than $\omega$ then $S_{i}$ and $S_{j}$ are identified as different surfaces.

\subsection{Experimental results}

Experimental results on six synthetic range images, each containing occluded surfaces, are presented. All the synthetic images are of size $128 \times 128$ and are shown in figures $7 \mathrm{a}-7 \mathrm{f}$. These images are referred to as image-1, image-2, image- 3 , image-4, image- 5 and image- 6 respectively. Boundaries of the surface patches in these images are shown in figures $8 \mathrm{a}-\mathrm{f}$ respectively, where each surface region is marked with an integer. Each of these images contain one or more occluded surface patches. The fuzzy classification results are shown in table 6 . The table shows the original class and the original parameters of the surfaces, the dominant class of the fitted surfaces and the estimated parameters when surfaces of respective dominant classes are fitted.

From table 6 it is seen that the occluded surfaces, i.e., the cone in image-1 (figure 7a), the cylinder in image- 2 (figure $7 \mathrm{~b}$ ) and the sphere in image- 3 (figure $7 \mathrm{c}$ ), are correctly classified and the estimated parameters of the fitted surfaces closely approximate the original parameters. Image-4 (figure 7d) contains two partially visible hemispherical surfaces (surface patches 2 and 4 in figure $8 \mathrm{~d}$ ), one with radius 55 and the other with radius 30 . Both of them have the same centre $(63,63,150)$. Both these hemispherical surfaces are correctly classified and parametrized individually. But since the region of occurrence of one hemisphere does not intersect that of the other, they are not considered as part of a single surface. Whereas in image-5 (figure 7e), though the regions of occurrence of two partially occluded hemispheres (surface 2 with radius 50 and surface 4 with radius 55 in figure 8 e) intersect, the combined surface shows conical behaviour though individually they are dominantly spherical. Hence they also cannot comprise a single surface. Two partially visible conical surface patches ( 2 and 4 in figure $8 f$ ) in image-6 (figure $7 f$ ) together construct a single conical surface whose estimated parameters closely approximate the estimated parameters of the individual surfaces and also those of the original surface. The cylindrical surfaces in all these three images are correctly classified and parametrized.

\section{Conclusion}

In this paper various geometric methods for surface fitting and surface characterization have been considered. Geometric surface fitting techniques give best results when the input is from the restricted domain of plane, sphere, cylinder and cone. The problem 


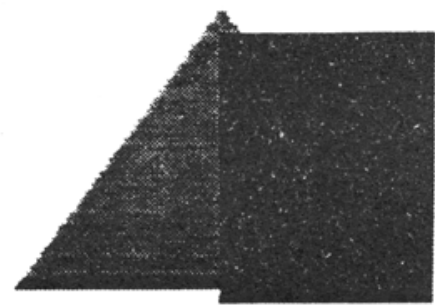

(a)

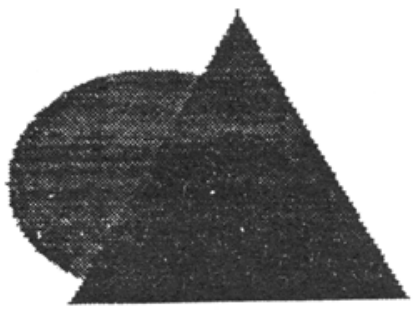

(c)

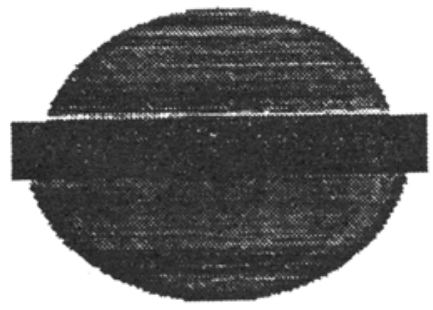

(e)

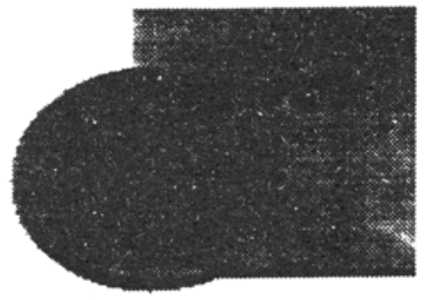

(b)

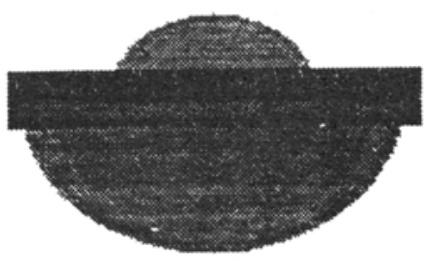

(d)

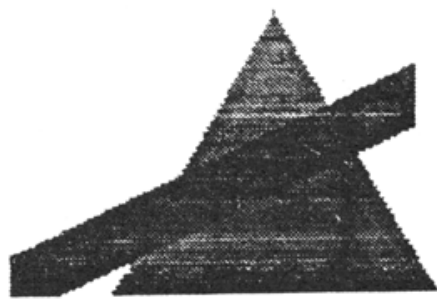

(f)

Figure 7. Synthetic range images containing occluded surfaces: (a) Image-1, (b) image-2, (c) image-3, (d) image-4, (e) image-5, and (f) image-6.

has been attempted for two different situations. In the first case, it has been assumed that a priori knowledge about the shape of the surface is available. In such situations, a surface of the proper type is fitted to the set of points representing the surface patch and the parameters of the fitted surface are extracted. This strategy of selective surface fitting reduces the amount of computation.

In the second case, when no a priori information regarding the shape of the unknown surface is available, the characterization of the surface is obtained in the form of a fuzzy membership vector. The unknown surface is taken as belonging to the class with maximum membership value. In this case the burden of fitting all four classes of surfaces cannot be avoided. The design of the fuzzy classifier plays a crucial role in determining the performance of the system. The performance of the classification scheme depends upon how correctly it can classify unknown surfaces. Thus the choice 


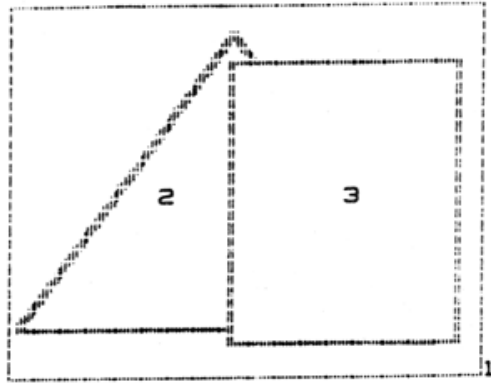

(a)

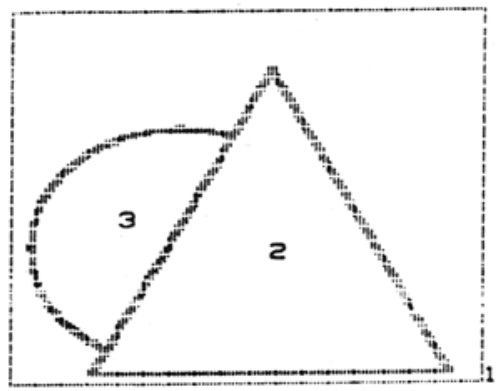

(c)

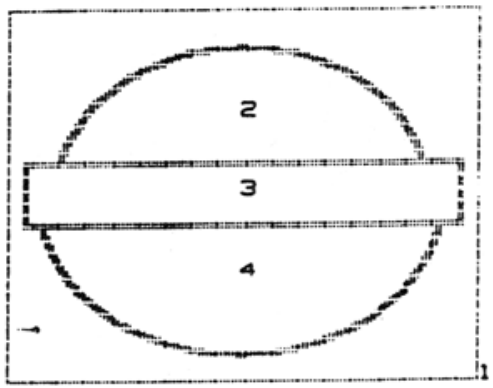

(e)

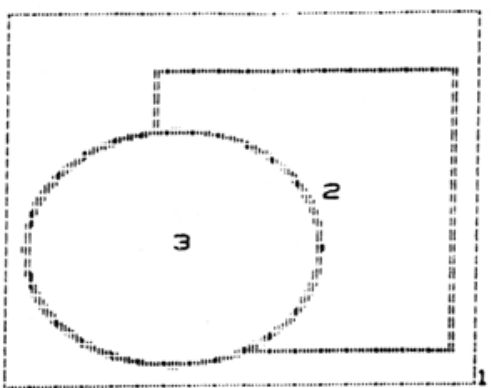

(b)

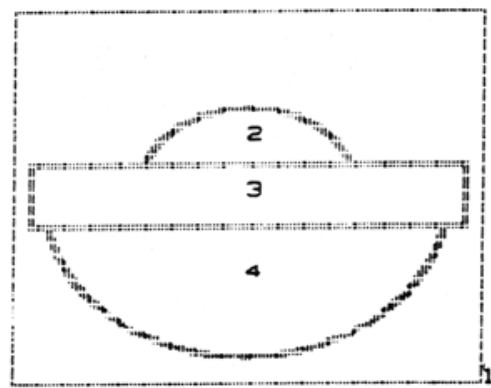

(d)

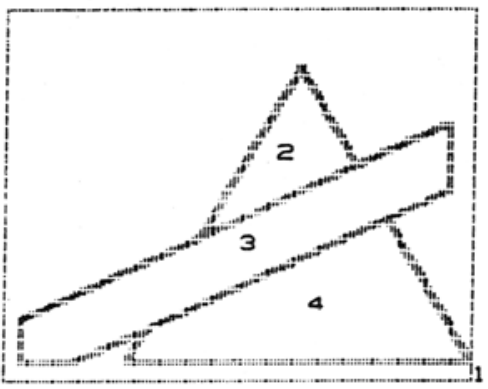

(f)

Figure 8. Boundaries of surface patches in synthetic range images: (a) Image-1, (b) image-2, (c) image-3, (d) image-4, (e) image-5, and (f) image-6.

of training samples to design the classifier is also very important. Experimental results on partially visible surfaces indicate that the geometric method for surface fitting along with the fuzzy classification scheme forms a reliable tool for handling occlusion. 


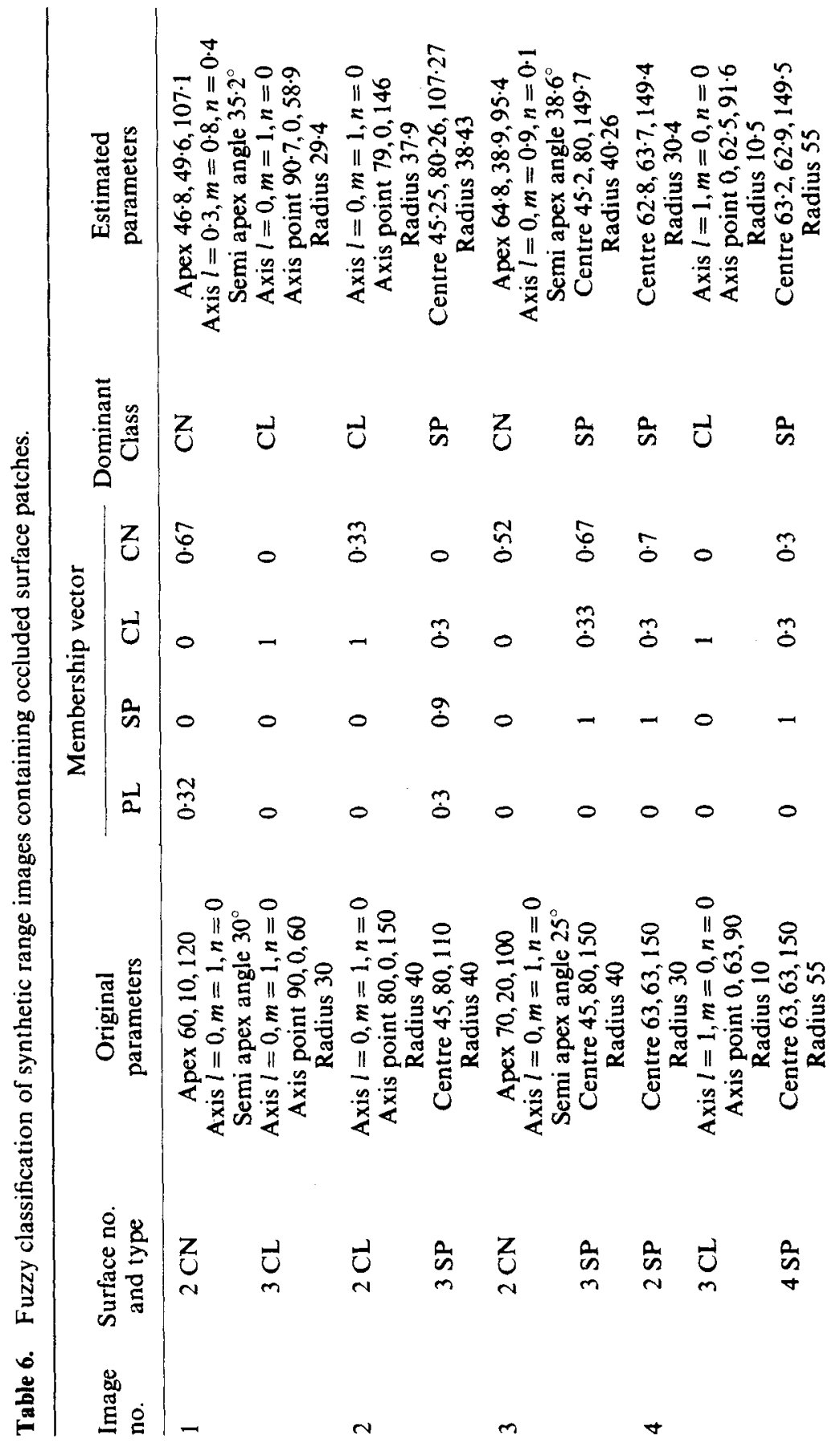




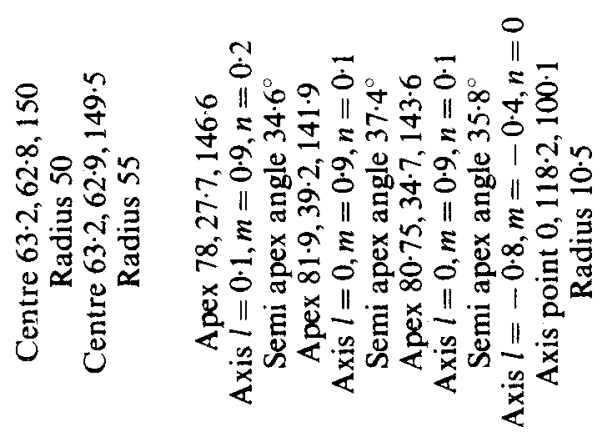

的云孞元它它

$\dot{0} \hat{0} \tilde{\theta} \hat{0} \dot{0} \hat{\delta} \quad \hat{0}$

$\hat{\theta} \ddot{0} \ddot{0} 0 \quad \hat{0} 0-$

- - ino ஸ̂ 00

0000000

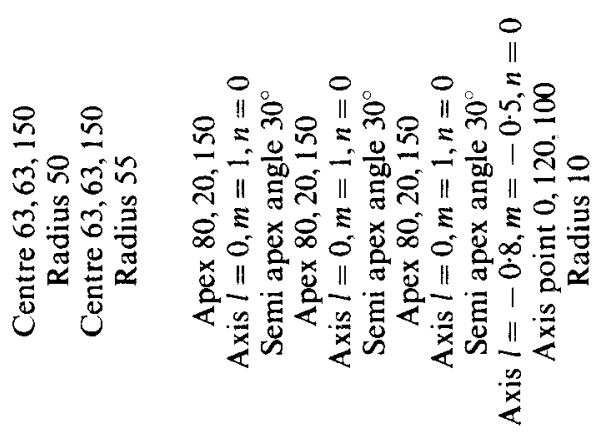

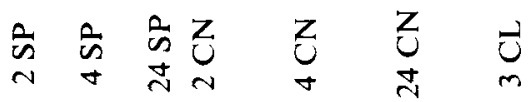


The authors are grateful to one of the reviewers for his/her valuable comments on an earlier version of this paper.

\section{References}

Besl P J, Jain R C 1984 Surface characterization for three dimensional object recognition. RSD-TR-20-84, Electrical Eng. and Computer Science Dept. University of Michigan, Ann Arbor, Michigan

Besl P J, Jain R C 1988 Segmentation through variable order surface fitting. IEEE Trans. Pattern Anal. Mach. Intell. 10: 167-192

Biswas P K, Mukherjee J, Chatterji B N 1990 Segmentation of range images. Proc. Workshop on Signal Processing. Communications and Networking, Indian Inst. Sci., Bangalore, 123-130

Bolle R M, Cooper D B 1984 Bayesian recognition of local 3-D shape by approximating image intensity functions with quadric polynomials. IEEE Trans. Pattern Anal. Mach. Intell. 6: $418-429$

Brady M, Ponce J, Yuille A, Asada H 1985 Describing surfaces. Comput. Vision Graph. Image Process. 32: 1-28

Faugeras O D 1984 New steps toward a flexible 3-D vision system for robotics. Int. Conf. on Pattern Recognition 2: 796-805

Han J H, Volz R A, Mudge T H 1987 Range image segmentation and surface parameter extraction for 3-D object recognition of industrial parts. Proc. IEEE Int. Conf. on Robotics and Automation 1: 380-386

Lin X, Wee W G 1985 Shape detection using range data. IEEE Int. Conf. on Robotics and Automation pp. 34-39

Muller Y, Mohr R 1984 Planes and quadrics detection using Hough transform. 7th Int. Conf. on Pattern Recognition pp 1101-1103

Shanti Narayan 1985 Analytical solid geometry (New Delhi: S Chand)

Taylor R W, Savini M, Reeves A P 1989 Fast segmentation of range imagery into planar regions. Comput. Vision Graph. Image Process. 45: 42-60 